\title{
Implications and Limits of Sequences
}

\author{
Alexandre Costa-Leite \\ University of Brasilia, Brasilia \\ Brazil \\ email: costaleite@unb.br \\ Edelcio G. de Souza \\ University of São Paulo, São Paulo \\ Brazil
}

email: edelcio.souza@usp.br

Abstract: This paper analyzes the problem of implication and attempts to characterize conditionals by a criterion of adequacy. A definition of implication based on the notion of limit of an infinite sequence is proposed.

Keywords: implications, conditionals, conjunctive limit, disjunctive limit

\section{Introduction}

Conditionals (or implications) are important operators especially because they are connected, in many cases, with consequence relations, as displayed in the famous deduction theorem. A conditional, at least in classical propositional logic, is a binary truth-function usually defined on a set $\{\mathrm{T}, \mathrm{F}\}$ of truth-values where $T$ refers to truth and $F$ refers to falsity. More precisely, semantically speaking, a truth-function $f:\{T, F\} \times\{T, F\} \rightarrow\{T, F\}$ is a classical implication if, and only if,

$$
f(T, T)=T ; f(T, F)=F ; f(F, T)=T, f(F, F)=T .
$$

This connective (i.e. material implication) is a bivalent binary truth-functional operator. But these properties hardly define what an implication is because there are connectives which deserve to be called implications and which cannot be explicitly defined using a bivalent semantical characterization, as the case of some implications in many-valued logics. Moreover, there are also non-truth-functional implications as the case of strict and discussive implications. Still, one can find implications with a 
behavior not based on truth-values, as the case of intuitionistic and linear implication. Therefore, it seems essential to find a criterion of adequacy able to determine when a given binary operator is an implication. This criterion, of course, is not able to determine univocally and describe precisely what an implication is, but it can, at least, guide us in discovering some clues about what implications really are, and which properties these connectives are supposed to have.

A study concerning the nature of implication is rather relevant, assuming that this operator is connected to the very essence of logic. Besides being a crucial logical connective, conditionals are often found in formulations of central notions in science and mathematics. By the way, some theorems are stated in an implicative form: the fundamental theorems of arithmetic, algebra and calculus could all be expressed by conditionals.

In this paper, we give two contributions: first, we use the notion of inferential systems to suggest a criterion of adequacy for conditionals. Then, we use limits of infinite sequences, a simple notion from mathematical analysis, to define implication.

\section{Inferential Systems and Conditionals}

The word implication is understood as something which refers to linguistic entities. Informally: implication is an operation in an algebra of propositions. More specifically, if $\mathbf{P}$ is a set whose elements are called sentences, then an implication is something like a function $f: \mathbf{P} \times \mathbf{P} \rightarrow \mathbf{P}$ such that for each pair $\langle p, q\rangle$ of elements of $\mathbf{P} \times \mathbf{P}$ we get an element $f\langle p, q\rangle$ of $\mathbf{P}$ denoted by $p \rightarrow q$. There is, however, an ambiguity that should be explained. We use the word implication to refer to the function $f$ as well as to elements of the image of $f$. In the first case, we say that $f$ is the symbol of implication and in the second case $(p \rightarrow q)$ is the implication of the sentence $q$ by $p$.

Assuming all above, a problem naturally arises: what are the conditions for a function of the type $f: \mathbf{P} \times \mathbf{P} \rightarrow \mathbf{P}$ be legitimately qualified as an implication? This is a question which is at the heart of what we call the problem of implication: how to legitimately characterize an implication? Indeed, this question is not an original one. Dunn and Hardegree ask in [3] the following version of the problem examined in this paper: "What are the (minimum) properties of a lattice operation, in virtue of which it is deemed an implication operation?" (p. 106). In a nutshell, given a complemented lattice, they answer this question providing the following requirements for an operation to be qualified as an implication ([3], pp. 105-109):

- Implication is a binary operation: they use a lattice, so implication is a partial order;

-(c1) (Truth) $p \leq q$ iff $p \rightarrow q=1$;

- (c2) (Modus Ponens) $p \wedge(p \rightarrow q) \leq q$;

-(c3) (Modus Tollens) $-q \wedge(p \rightarrow q) \leq-p$;

-(c4) (Refutation) $p \wedge-q \leq-(p \rightarrow q)$

Concerning these criteria, they state that: "Conditions (c1)-(c4) are collectively referred to as the minimal implicative conditions: every implication operation should satisfy all four conditions on any lattice with complementation." ([3], p. 108). Let's call this the algebraic solution to the problem of implication. Although it is very elegant to define implication algebraically, the fact that complement is used commits the solution with extra entities which could be avoided in order to define implication. In our opinion, to use forms of negation to define properties an implication should satisfy is not essential.

The algebraic solution to the problem of implication is dependent of complements (i.e. negations). So, it is not pure. A pure solution is provided by Koslow who looks for conditions on implications in [4] without appealing to extra logical entities. Koslow's idea consists in adopting an 
implication structure as a pair $\langle\mathbf{S}, \Rightarrow\rangle$ such that $\mathbf{S}$ is not empty and the implication relation $\Rightarrow$ is defined as $\Rightarrow \subseteq \wp^{<\infty}(\mathbf{S}) \times \mathbf{S}$, where $\wp^{<\infty}(\mathbf{S})$ denotes finite subsets of $\mathbf{S}$, and it obeys some laws reproduced below ([4], p. 5):

- (Projection) $p_{1}, \ldots, p_{n} \Rightarrow p_{k}$, for any $k=1, \ldots, n$;

- (Simplification) If $p_{1}, p_{1}, \ldots, p_{n} \Rightarrow q$, then $p_{1}, \ldots, p_{n} \Rightarrow q$;

- (Permutation) If $p_{1}, \ldots, p_{n} \Rightarrow q$, then $p_{f_{1}}, \ldots, p_{f_{n}} \Rightarrow q$, for any bijection $f$ of $\{1,2,3, \ldots, n\}$;

-(Cut) If $p_{1}, \ldots, p_{n} \Rightarrow q$ and $q, q_{1}, \ldots, q_{m} \Rightarrow r$, then $p_{1}, \ldots, p_{n}, q_{1}, \ldots, q_{m} \Rightarrow r$.

Koslow uses, in fact, six properties, but he argues that two other conditions, (Reflexivity) and (Dilution), can be derived from the properties above. He makes no use of any connective to define an implication relation. Structuralist logic proposed by Koslow assumes that "...implication is central to logic, and that the logical operators, as well as modal operators generally, can be characterized as such, by the way they interact with respect to implication."([5], p.167). Many logical structures such as the Tarskian consequence operator can be viewed as particular cases of implication structures (see [4], p.43). The structuralist theory of logic is so general in its understanding of what a logic is that it clearly anticipates what is currently known as universal logic (a concept developed in [2]). The notion of implication structure is used to define hypotheticals (i.e. Koslow's terminology for conditionals) (see [4], p.77-78). These are obtained from an implication structure if, and only if, two conditions are respected: the first one is modus ponens and the second one is the deduction theorem. This is Koslow's criterion of adequacy for conditionals. Let's call this the structural solution to the problem of implication.

Koslow's solution sounds better than that of Dunn and Hardegree, given that it is negation-free. Despite this fact, Koslow's answer to the problem of implication is committed to many general abstract inference rules, as he imposes many properties on implication relations. We think that something simpler can be obtained. Moreover, note that such a problem of implication could be approached at least in two different ways. From one side, we could search for a descriptive solution to the problem indicating the way in which implication is, in fact, characterized in language, and this seems to be the approach of Dunn and Hardegree, and also that of Koslow. They both try to describe what implications really are (descriptive solutions are, therefore, always open to refutations, since one can show they are false). From the other side, we could search for a normative solution showing how an implication should be characterized in a language. This kind of normative solution can only be properly judged by its utility, and not for its correctness. In a different way, we look for a normative solution, as we believe descriptive solutions have limits which cannot be transposed.

In order to present a proposal to the problem of implication we consider another concept strongly related to that of implication. It is the general concept of inferential system. With the aim of proposing a legitimate use of the concept of implication, we have to understand how it behaves with respect to certain admissible inferences inside an inferential system. This concept can be introduced from a general viewpoint.

An inferential system is a pair $\langle\mathbf{P}, \mid-\rangle$ such that $\mathbf{P}$ is a set of sentences and $\mid-$ is a consequence relation defined in $\wp(\mathbf{P}) \times \mathbf{P}$. Elements of $\mid-$ are, therefore, pairs $\langle\Gamma, \varphi\rangle$ such that $\Gamma$ is a subset of $\mathbf{P}$ while $\varphi$ is an element of $\mathbf{P}$. To point out that $\langle\Gamma, \varphi\rangle$ is a member of $\mid-$ the notation $\Gamma \mid-\varphi$ is used, and we say that $\varphi$ is a consequence of $\Gamma$ in $\langle\Gamma, \varphi\rangle$. We proceed as in [1]. So, we do not need to specify any kind of property that $\mid$ - should satisfy. Then, we can now introduce a proposal showing how to characterize implications inside inferential systems. The adequacy criterion for implications suggested is 
the following: Let $\langle\mathbf{P}, \mid-\rangle$ be an inferential system. A function $f: \mathbf{P} \times \mathbf{P} \rightarrow \mathbf{P}$, $\langle p, q\rangle \mapsto f(p, q)=(p \rightarrow q)$ is an implication with respect to $\langle\mathbf{P}, \mid-\rangle$ if, and only if, the following conditions are satisfied, for all subsets $\Gamma$ of $\mathbf{P}$ and sentences $p$ and $q$ :

- Rule of modus ponens (MP): if $\Gamma \mid-f(p, q)$ and $\Gamma \mid-p$, then $\Gamma \mid-q$;

- Rule of reflexivity $(\mathrm{R}): \Gamma \mid-f(p, p)$.

- Deduction theorem (DT): $\Gamma, p \mid-q$ iff $\Gamma \mid-f(p, q)$

Note that the criteria above are sufficiently broad to capture cases in which $\mid-$ is defined semantically, syntactically or in any other specific way. An implication is perfect if, and only if, besides validating (MP) and (R), it satisfies (DT). A degenerated implication is any implication which does not satisfy all conditions above. And, for us, an ideal implication is a perfect implication. In classical logic, it is easy to check that there is an operator which is a perfect implication: material implication clearly satisfies both (MP), (R) and (DT). A few comments on these conditions are called for. (MP) sounds essential for implication because it deals with truth-preservation, be it semantical or syntactical, and (MP) alone cannot define implication. (R) assures that neither conjunctions nor disjunctions are implications, and this cannot be assured only by an operator satisfying (MP). The deduction theorem sounds important as it connects implication with the central notion of logic, i.e., logical consequence.

Our solution looks very similar to that proposed by Koslow, with some important distinctions: Implication structures are committed to many properties on the implication relation, while our inferential system does not have any property at all. So, inferential systems are much more general. And we also impose reflexivity as a condition, while Koslow's criterion uses only (MP) and (DT).

Before introducing the main contribution of this paper, that is, the definition of implication by means of limits of infinite sequences, let's note that there are in the literature, however, many sorts of conditionals. This is a remarkable list: material, strict, counterfactual, linear, discussive, a great variety of many-valued conditionals and so on. Are all these perfect implications according to the criterion to determine whether a binary relation is or not an implication? Certainly not. In the same way that there are negations which are not truth-functional and which behavior is totally different from classical negation, we still call such operators negations. It would be a dogmatic attitude just to say that they are not negations because they do not behave like classical negation. The same situation happens here. Some implications are not like classical implication, and they do violate our criterion of adequacy, but they still deserve to be called implications, although they are not perfect implications.

\section{Implications as Limits of Sequences}

The next step we intend to develop is to get a notion of implication based on the notion of limit as it is used in mathematical analysis. Consider an inferential system $\langle\mathbf{P}, \mid-\rangle$ such that $\mathbf{P}$ is a set of formulas and $\mid-$ is a consequence relation. Be

$$
\left(p_{i}\right)_{i \in \mathrm{N}}=p_{0}, p_{1}, \ldots, p_{i}, \ldots
$$

a sequence of elements of $\mathbf{P}$. So we propose the following definitions:

Definition 3.1 (Conjunctive limit) We say that $p$ is the conjunctive limit of $\left(p_{i}\right)_{i \in \mathrm{N}}$ 


$$
\lim _{i \rightarrow \infty}^{c} p_{i}=p
$$

if, and only if, $p \mid-p_{i}$, for all $i \in \mathrm{N}$.

Definition 3.2 (Disjunctive limit) We say that $p$ is the disjunctive limit of $\left(p_{i}\right)_{i \in \mathrm{N}}$

$$
\lim _{i \rightarrow \infty}^{d} p_{i}=p
$$

if, and only if, $p_{i} \mid-p$, for each $i \in \mathrm{N}$.

Consider now two sequences $\left(p_{i}\right)_{i \in \mathrm{N}}$ and $\left(q_{i}\right)_{i \in \mathrm{N}}$ such that conjunctive and disjunctive limits do exist respectively. Assume that there are sequences $\left(p_{i} \wedge q_{i}\right)_{i \in \mathrm{N}}$ and $\left(p_{i} \vee q_{i}\right)_{i \in \mathrm{N}}$.

Theorem 3.3 The following holds:

$$
\begin{aligned}
& \lim _{i \rightarrow \infty}^{c}\left(p_{i} \wedge q_{i}\right)=\lim _{i \rightarrow \infty}^{c} p_{i} \wedge \lim _{i \rightarrow \infty}^{c} q_{i} \\
& \lim _{i \rightarrow \infty}^{d}\left(p_{i} \vee q_{i}\right)=\lim _{i \rightarrow \infty}^{d} p_{i} \vee \lim _{i \rightarrow \infty}^{d} q_{i}
\end{aligned}
$$

The proof follows from the definitions of conjunctive and disjunctive limits. It is straightforward to show that De Morgan's laws and classical material implication can be defined in this framework. To see this, we define the negation of a sequence $\left(p_{i}\right)_{i \in \mathrm{N}}$, in symbols, $\neg\left(p_{i}\right)_{i \in \mathrm{N}}$ as the sequence given by $\left(\neg p_{i}\right)_{i \in \mathrm{N}}$. Then, if the negation is the classical one, it is easy to see that:

Theorem 3.4 The following holds:

$$
\begin{aligned}
& \lim _{i \rightarrow \infty}^{c}\left(\neg p_{i}\right)=\neg \lim _{i \rightarrow \infty}^{d} p_{i} \\
& \lim _{i \rightarrow \infty}^{d}\left(\neg p_{i}\right)=\neg \lim _{i \rightarrow \infty}^{c} p_{i}
\end{aligned}
$$

With negations at hands, it is easy to see how to define material implication. However, we do not want to use negation. So, now, in order to define what an implication is (without using classical negation), consider two propositions $p$ and $q$.

Definition 3.5 We say that $p$ implies $q$ (i.e. $p \rightarrow q$ ), if $q$ is the disjunctive limit of $a$ sequence in which $q$ occurs. Therefore, $p \rightarrow q$ if, and only if, there exists a sequence $\left(q_{n}\right)_{n \in \mathrm{N}}$ such that

$$
\lim _{n \rightarrow \infty}^{d}\left(q_{n}\right)=q
$$


and $p=q_{i}$, for some $i \in \mathrm{N}$.

This implication satisfies the three conditions (MP), (R) and (DT) exposed above. We prove that it satisfies (DT):

Theorem 3.6 $p \mid-q$ if, and only if, $p \rightarrow q$.

Proof. Assume first that $p \mid-q$. Take the sequence $\left(q_{n}\right)_{n \in \mathrm{N}}$ with $q_{n}=q$ for all $n \in \mathrm{N}$. In this case,

$$
\lim _{n \rightarrow \infty}\left(q_{n}\right)=q
$$

and, thus, $p \rightarrow q$. Now, assume that $p \rightarrow q$. Then, there exists a sequence $\left(q_{n}\right)_{n \in \mathrm{N}}$ with

$$
\lim _{n \rightarrow \infty}^{d}\left(q_{n}\right)=q
$$

and $q_{i}=p$, for some $i \in \mathrm{N}$. Thus, $q_{i} \mid-q$, i.e, $p \mid-q$. Q.E.D.

The original notions of conjunctive and disjunctive limits help us to define what an implication is from a general perspective, without using any other connective, in the same spirit of Koslow's Structuralist Logic.

\section{Conclusion}

We saw that the algebraic solution to the problem of implication has unnecessary commitments as it uses the notion of complementation (i.e. negation). We also saw that the structural solution is not too general and, by the end, implication relation is a sort of consequence relation rather than a connective. It is possible, despite this fact, to define implication as a connective and Koslow imposes only two conditions on it: (MP) and (DT). So, according to our viewpoint, hypotheticals are degenerated conditionals. These solutions are descriptive and they try to answer the question of what an implication really is.

As we have seen, our normative criterion of adequacy for conditionals sounds efficient as it is captures in a general and free mode some relevant properties implications should satisfy. As we believe, these are the main features for classifying an implication. From one side, a descriptive criterion is very difficult to provide, as it should be a criterion able to describe precisely all kinds of conditionals. On the other side, a normative criterion is feasible and it plays a pragmatic role.

By the end, we undertook an attempt to model logical connectives, and especially implication, using the notion of limits of infinite sequences. This is the main contribution here, and it is based on a connection between mathematical analysis and logic.

\section{References}

1. Bensusan, H, Costa-Leite, A, De Souza, E. Logics and Their Galaxies. In. The Road to Universal Logic, vol.2, A. Koslow and A. Buchsbaum (eds.), Basel: Birkhäuser, 2015, pp.243-252.

2. Beziau, J-Y. Universal Logic, In. Logica 1994, T. Childers and O. Majer (eds.), Prague: Filosofia, 1994, pp. 73-93.

3. Dunn, M, Hardegree, G. Algebraic Methods in Philosophical Logic, Oxford: Oxford University Press, 2001. 
4. Koslow, A. A structuralist theory of logic, Cambridge: Cambridge University Press, 1992.

5. Koslow, A. Structuralist Logic: Implications, Inferences and Consequences, Logica Universalis, vol. 1, 2007, pp. 167-181. 